\title{
Effects of lithium on oxidative stress parameters in healthy subjects
}

\author{
RUSHANIYA KHAIROVA ${ }^{1,3^{*}}$, ROHIT PAWAR ${ }^{3 *}$, GIACOMO SALVADORE $^{1}$, MARIO F. JURUENA $^{5}$, \\ RAFAEL T. DE SOUSA ${ }^{2}$, MÁRCIO G. SOEIRO-DE-SOUZA ${ }^{2}$, MIRIAN SALVADOR ${ }^{4}$, \\ CARLOS A. ZARATE ${ }^{1}$, WAGNER F. GATTAZ ${ }^{2}$ and RODRIGO MACHADO-VIEIRA ${ }^{1,2}$ \\ ${ }^{1}$ Experimental Therapeutics, Mood and Anxiety Disorders Program, National Institute of Mental Health, \\ National Institutes of Health, Bethesda, MD 20892, USA; ${ }^{2}$ Laboratory of Neuroscience, LIM-27, Institute and \\ Department of Psychiatry, University of Sao Paulo, SP, Brazil; ${ }^{3}$ Department of Psychiatry, Maimonides Medical \\ Center, New York, NY, USA; ${ }^{4}$ Institute of Biotechnology, University of Caxias do Sul, Caxias do Sul, RS; \\ ${ }^{5}$ Department of Neuroscience and Behavior, University of Sao Paulo, Ribeirao Preto, Brazil
}

Received September 6, 2011; Accepted December 6, 2011

DOI: $10.3892 / \mathrm{mmr} .2011 .732$

\begin{abstract}
Increased neuronal oxidative stress $(\mathrm{OxS})$ induces deleterious effects on signal transduction, structural plasticity and cellular resilience, mainly by inducing lipid peroxidation in membranes, proteins and genes. Major markers of OxS levels include the thiobarbituric acid reactive substances (TBARS) and the enzymes superoxide dismutase (SOD), catalase (CAT) and glutathione peroxidase. Lithium has been shown to prevent and/or reverse DNA damage, free-radical formation and lipid peroxidation in diverse models. This study evaluates OxS parameters in healthy volunteers prior to and following lithium treatment. Healthy volunteers were treated with lithium in therapeutic doses for 2-4 weeks. Treatment with lithium in healthy volunteers selectively altered SOD levels in all subjects. Furthermore, a significant decrease in the SOD/ CAT ratio was observed following lithium treatment, wich was associated with decreased $\mathrm{OxS}$ by lowering hydrogen peroxide levels. This reduction in the SOD/CAT ratio may lead to lower $\mathrm{OxS}$, indicated primarily by a decrease in the concentration of cell hydrogen peroxide. Overall, the present findings indicate a potential role for the antioxidant effects of lithium in healthy subjects, supporting its neuroprotective profile in bipolar disorder (BD) and, possibly, in neurodegenerative processes.
\end{abstract}

Correspondence to: Dr Rodrigo Machado-Vieira, Institute and Department of Psychiatry, LIM-27, Rua Ovidio Pires de Campos, 785, 01060-970 Sao Paulo, SP, Brazil

E-mail: rvieira@usp.br; machadovieirar@gmail.com

${ }^{*}$ Contributed equally

Abbreviations: BD, bipolar disorder; CAT, catalase; OxS, oxidative stress; SOD, superoxide dismutase; TBARS, thiobarbituric acid reactive substances

Key words: lithium, oxidative stress, healthy, superoxide dismutase, bipolar disorder

\section{Introduction}

Increased neuronal oxidative stress $(\mathrm{OxS})$ induces deleterious effects on signal transduction, structural plasticity and cellular resilience, mostly by inducing lipid peroxidation in membranes, proteins and genes $(1,2)$. It has been hypothesized that these pathological processes occur in critical brain circuits that regulate affective functioning, emotions, motoric behavior and pleasure involved in bipolar disorder (BD) (3).

Altered OxS parameters have been described in the pathophysiology and therapeutics of $\mathrm{BD}(2,4)$. The brain is particularly vulnerable to oxidative damage since it contains large amounts of polysaturated fatty acids and possesses low antioxidant capacity $(5,6)$.

Major markers of OxS levels include the thiobarbituric acid reactive substances (TBARS) and the enzymes superoxide dismutase (SOD), catalase (CAT) and glutathione peroxidase (7). SOD levels typically decrease when stress conditions are reduced. By contrast, elevated SOD/CAT ratio induces an increase in OxS levels, mostly associated with elevation in cell hydrogen peroxide concentration (8).

Lithium is the gold standard mood stabilizing agent that has been found to prevent and/or reverse DNA damage, freeradical formation and lipid peroxidation in diverse models (9-11). Lithium plays a neurotrophic and neuroprotective role and enhances total antioxidant activity in diverse models $(4,9,11,12)$. In BD, lithium treatment significantly reduces the levels of plasma lipid peroxides and improves antioxidant status $(2,10)$. Lithium has also been shown to decrease SOD levels in pre-clinical models compared to animals submitted to an animal model of mania (amphetamine) in the prefrontal cortex (13).

The emerging body of data presenting the antioxidant and neuroprotective effects of lithium warrants further investigation in identifying the specific targets that mediate these unique properties. However, few studies have examined the potential role of lithium as an antioxidant in non-pathological conditions. Thus, in this study we evaluated OxS parameters in healthy volunteers prior to and following lithium treatment. 


\section{Materials and methods}

Ten healthy volunteers were screened by two psychiatrists for medical and psychiatric disorders. All subjects were medically healthy, as determined by physical and neurological examinations and laboratory tests. Exclusion criteria included a current or past psychiatric condition (based on the Structured Clinical Interview for DSM-IV; 14), a first degree family history of psychiatric disorders (based on a family history screen), and a chronic medical condition. Use of anti-inflammatory agents, oral contraceptives, statins or herbal therapies was not permitted during the study. In addition, subjects were required to refrain from the use of alcohol, tobacco and caffeine for 1 month prior to the study. The study was approved by the local Ethics Committee and all subjects provided written informed consent before participating in the study.

The follow-up period ranged from 2-4 weeks (mean duration 15.2 \pm 6.1 days) (Fig. 1). Subjects started with $300 \mathrm{mg} /$ day, which was subsequently increased to $600 \mathrm{mg} /$ day or more until they reached therapeutic levels $(0.6-1 \mathrm{mM} / 1)$. Two subjects used lithium for 6 and 9 days and did not conclude 2 weeks of therapy due to side effects reported (nausea; $n=2$ ).

Samples were collected in the morning, 10-12 $\mathrm{h}$ after the last lithium dose. Blood samples were obtained using vacutainer tubes and kept on ice. Samples were centrifuged at $3,000 \mathrm{x} \mathrm{g}$ for $15 \mathrm{~min}$ and stored at $-80^{\circ} \mathrm{C}$ for $<6$ months. All samples and standards were assessed in duplicate. SOD activity (U/g) was determined spectrophotometrically by inhibition of the autocatalytic adrenochrome formation rate at $480 \mathrm{~nm}$, which was measured using $1 \mathrm{mmol} / 1$ adrenaline $(\mathrm{pH} 2.0)$ and $50 \mathrm{mmol} / \mathrm{l}$ glycine $(\mathrm{pH}$ 10.2). The reaction was conduced at $30^{\circ} \mathrm{C}$ during $3 \mathrm{~min}$ (15). CAT assay was carried out accordingly (16). Total serum protein levels were evaluated using the Total Proteins kit from Labtest ${ }^{\circledR}$. TBARS levels were measured on the production of MDA, which, when combined with thiobarbituric acid, forms a pink chromogen compound (17).

Statistical analysis was performed using SPSS 14. Results are presented as the means \pm standard deviation. Paired t-test (two-tailed) was used to determine the difference between the two groups. Statistical significance was set at $\mathrm{p}<0.05$ (Bonferroni correction). The correlation was studied by linear regression analysis and $\mathrm{r}$ values were calculated by the Pearson test.

\section{Results}

Subjects ( 3 males/7 females) were on average $23.3 \pm 3$ years of age. The mean serum lithium levels and dose achieved during the study were $0.85 \pm 0.22 \mathrm{mM} / 1$ and $770 \pm 125 \mathrm{mg} / \mathrm{day}$, respectively. The mean duration of lithium treatment was $15.2 \pm 6.1$ days.

SOD levels in healthy volunteers significantly decreased after lithium treatment $(1.19 \pm 0.28 \mathrm{U} / \mathrm{g})$ compared to baseline levels (2.61 $\pm 0.51 \mathrm{U} / \mathrm{g}, \mathrm{p}=0.01)$. Lower SOD/CAT ratio was observed after treatment $(4.12 \pm 1.32)$ compared to baseline $(9.72 \pm 2.34$, $\mathrm{p}=0.02)$. By contrast, CAT levels were not significantly affected by lithium treatment (post-treatment $0.511 \pm 0.36 \mu \mathrm{mol} / \mathrm{mg}$ vs. pre-treatment $0.5 \pm 0.18 \mu \mathrm{mol} / \mathrm{mg} ; \mathrm{p}=0.48)$. Similarly, no significant changes were observed in TBARS levels following

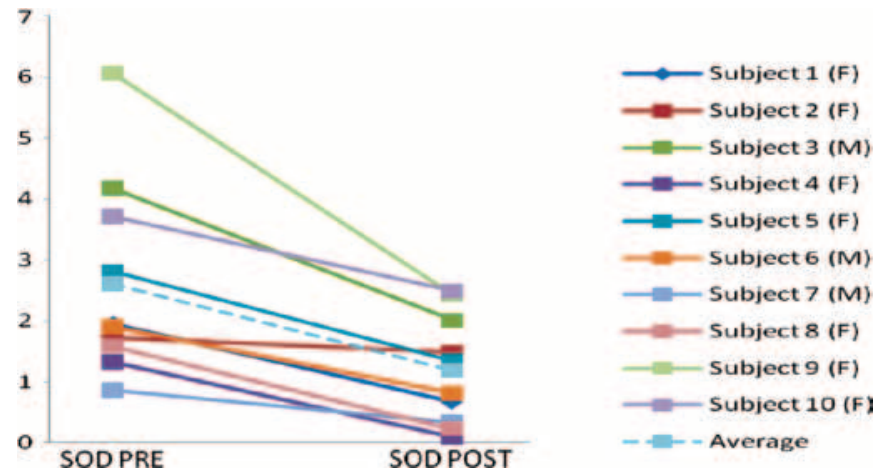

Figure 1. Plasma superoxide dismutase (SOD) and the ratio of SOD/catalase in healthy subjects pre- and post-lithium treatment (2-4 weeks). Results of SOD activity pre- and post-treatment are represented for each of the 10 healthy volunteers.

treatment with lithium $(6.93 \pm 0.58 \mathrm{nmol} / \mathrm{ml})$ as compared to baseline levels $(6.18 \pm 0.35 \mathrm{nmol} / \mathrm{ml} ; \mathrm{p}=0.14)$ (Fig. 1). No statistical difference was found between male and female subjects in SOD, CAT and TBARS levels when compared prior to and following treatment with lithium. Plasma lithium levels and duration of treatment were not correlated to any marker.

Lithium was generally well tolerated; the most common side effects were diarrhea $(n=3)$, nausea $(n=3)$, vomiting $(n=2)$, fatigue $(n=2)$, somnolence $(n=1)$ and constipation $(n=1)$. Mood enhancing effects were not observed during the trial (Young Mania Rating Scale scores). Two subjects were discontinued earlier in this study secondary to adverse effects (nausea and vomiting).

\section{Discussion}

To our knowledge, this is the first study to evaluate the specific effects of lithium on the OxS parameters of healthy subjects. The present study showed a selective decrease in SOD levels after lithium treatment in all healthy volunteers, inducing no effect on the lipid oxidation marker TBARS and the antioxidant enzyme CAT levels. SOD/CAT ratio significantly decreased in healthy volunteers after lithium treatment. This reduction in SOD/CAT ratio may lead to lower OxS, which is reflected mainly in a decrease in the concentration of cell hydrogen peroxide (8).

Several studies have demonstrated altered OxS parameters in the pathophysiology and therapeutics of $\mathrm{BD}$, including changes in SOD, CAT and TBARS levels $(2,10,18)$. Superoxide also generates hydrogen peroxide through a dismutation reaction catalyzed by SOD (19). In the present study, lithium was found to limit the enzyme activity, potentially lowering hydrogen peroxide and hydroxyl radical formation. Similarly, lithium was also shown to reverse increased OxS parameters in $\mathrm{BD}(2,20)$. For instance, a decline in lipid peroxidation and an increase in CAT levels were observed in lithium-treated rats $(9,21)$.

The potential effects of lithium on TBARS, SOD and CAT levels in subjects with BD (2), associated with the lack of modulatory effect in healthy subjects described in the present study, suggest that only regulation targeting TBARS and CAT (but not SOD) may be selectively associated with the thera- 
peutic effects of lithium in BD. In line with our findings, it has been suggested that changes in SOD levels are not always indicative of the presence of OxS per se (13). Furthermore, lithium decreases prefrontal cortex SOD levels compared to saline solution (13).

Certain limitations should be considered when interpreting the results. These include the small size and the short duration of the treatment with lithium. Nevertheless, it is important to note that lithium has been proven to induce significant antimanic effects in large clinical trials, even in the first 2 weeks of treatment $(22,23)$.

Overall, the present findings support a role for lithium's antioxidant actions, which appear more limited in healthy subjects than in individuals with BD. Our results support recent studies that reveal the key role of lithium in diverse neuropsychiatric disorders. However, further studies evaluating the specific effects of lithium on OxS markers and other molecular targets are still required.

\section{Acknowledgements}

The authors thank all the volunteers who participated in this study. Dr Machado-Vieira would like to thank the Stanley Medical Research Institute (SMRI), Sao Paulo Research Foundation (Fapesp, Sao Paulo, Brazil) and ABADHS (Associação Beneficente Alzira Denise Hertzog da Silva).

\section{References}

1. Mahadik SP, Evans D and Lal H: Oxidative stress and role of antioxidant and omega-3 essential fatty acid supplementation in schizophrenia. Prog Neuropsychopharmacol Biol Psychiatry 25: 463-469, 2001

2. Machado-Vieira R, Andreazza AC, Viale CI, et al: Oxidative stress parameters in unmedicated and treated bipolar subjects during initial manic episode: a possible role for lithium antioxidant effects. Neurosci Lett 421: 33-36, 2007.

3. Zarate CA, Singh J Jr and Manji HK: Cellular plasticity cascades: targets for the development of novel therapeutics for bipolar disorder. Biol Psychiatry 59: 1006-1020, 2006.

4. Andreazza AC, Kauer-Sant'Anna M, Frey BN, et al: Effects of mood stabilizers on DNA damage in an animal model of mania. J Psychiatry Neurosci 33: 516-524, 2008.

5. Floyd RA: Antioxidants, oxidative stress, and degenerative neurological disorders. Proc Soc Exp Biol Med 222: 236-245, 1999.

6. Halliwell B: Role of free radicals in the neurodegenerative diseases: therapeutic implications for antioxidant treatment. Drugs Aging 18: 685-716, 2001.
7. Wang JF, Azzam JE and Young LT: Valproate inhibits oxidative damage to lipid and protein in primary cultured rat cerebrocortical cells. Neuroscience 116: 485-489, 2003.

8. Gsell W, Conrad R, Hickethier M, et al: Decreased catalase activity but unchanged superoxide dismutase activity in brains of patients with dementia of Alzheimer type. J Neurochem 64: 1216-1223, 1995.

9. Shao L, Young LT and Wang JF: Chronic treatment with mood stabilizers lithium and valproate prevents excitotoxicity by inhibiting oxidative stress in rat cerebral cortical cells. Biol Psychiatry 58: 879-884, 2005.

10. Andreazza AC, Cassini C, Rosa AR, et al: Serum S100B and antioxidant enzymes in bipolar patients. J Psychiatr Res 41: 523-529, 2007

11. Machado-Vieira R, Manji HK and Zarate CA Jr: The role of lithium in the treatment of bipolar disorder: convergent evidence for neurotrophic effects as a unifying hypothesis. Bipolar Disord 11: 92-109, 2009.

12. Schafer M, Goodenough S, Moosmann B and Behl C: Inhibition of glycogen synthase kinase 3 beta is involved in the resistance to oxidative stress in neuronal HT22 cells. Brain Res 1005: 84-89, 2004.

13. Frey BN, Valvassori SS, Reus GZ, et al: Effects of lithium and valproate on amphetamine induced oxidative stress generation in an animal model of mania. J Psychiatry Neurosci 31: 326-332, 2006.

14. First MB and Gibbon RL: Structured Clinical Interview for DSM-IV-TR Axis I Disorders, Research Version, Non-patient Edition. (SCID-I/NP) New York: Biometrics Research, New York State Psychiatric Institute, 2002.

15. Mirsa HP and Fridovich I: The role of superoxide anion in the autoxidation of epinephrine and a simple assay for superoxide dismutase. J Biol Chem 217: 3170-3175, 1972.

16. Aebi H: Catalase in vitro. Methods Enzymol 105: 121-126, 1984.

17. Wills ED: Mechanisms of lipid peroxide formation in animal tissues. Biochem J 19: 667-676, 1966.

18. Ranjekar PK, Mahadik SP, Hegde MV, et al: Decreased antioxidant enzymes and membrane essential polyunsaturated fatty acids in schizophrenic and bipolar mood disorder patients. Psychiatry Res 121: 109-122, 2003.

19. Liochev SI and Fridovich I: The relative importance of HO* and. Free Radic Biol Med 26: 777-778, 1999.

20. Frey BN, Martins MR, Petronilho FC, Dal-Pizzol F, Quevedo J and Kapczinski F: Increased oxidative stress after repeated amphetamine exposure: possible relevance as a model of mania. Bipolar Disord 8: 275-280, 2006.

21. Tandon A, Dhawan DK and Nagpaul JP: Effect of lithium on hepatic lipid peroxidation and antioxidative enzymes under different dietary protein regimens. J Appl Toxicol 18: 187-190, 1998.

22. Machado-Vieira R, Soares JC, Lara DR, et al: A double-blind, randomized, placebo-controlled 4-week study on the efficacy and safety of the purinergic agents allopurinol and dipyridamole adjunctive to lithium in acute bipolar mania. J Clin Psychiatry 69: 1237-1245, 2008

23. Bowden CL, Brugger AM, Swann AC, et al: Efficacy of divalproex vs lithium and placebo in the treatment of mania. The Depakote Mania Study Group. JAMA 271: 918-924, 1994. 